\title{
Transcatheter trans-septal mitral valve-in-valve implantation
}

\author{
Vivian G. $\mathrm{Ng}^{1}$, Susheel Kodali ${ }^{1}$, Isaac George ${ }^{2}$ \\ ${ }^{1}$ Division of Interventional Cardiology, ${ }^{2}$ Division of Cardiothoracic Surgery, New York Presbyterian Hospital, Columbia University Medical Center, \\ New York, NY, USA \\ Correspondence to: Isaac George, MD. Division of Cardiothoracic Surgery, New York Presbyterian Hospital, Columbia University Medical Center, \\ 177 Fort Washington Ave. MHB 7GN-435, New York, NY 10032, USA. Email: ig2006@columbia.edu.
}

Submitted Jul 09, 2018. Accepted for publication Sep 03, 2018.

doi: $10.21037 /$ acs.2018.09.04

View this article at: http://dx.doi.org/10.21037/acs.2018.09.04

\section{Clinical vignette}

Our patient is 74-year-old man with a history of hypertension, hyperlipidemia, prior stroke, paroxysmal atrial fibrillation (on anticoagulation) who had a prior bioprosthetic mitral valve replacement (\#33 St. Jude Medical Epic), tricuspid valve repair (\#34 Contour Ring) who was doing well until 2 months prior to presentation when he noted increasing shortness of breath with exertion, lower extremity edema and weight gain. His symptoms progressed despite diuresis. On transthoracic echocardiography, he was noted to have depressed left ventricular (LV) function (ejection fraction $45 \%$ ) and moderate to severe mitral regurgitation of his bioprosthetic mitral valve. His Society of Thoracic Surgeons (STS) score for a re-operative surgical mitral valve replacement was $6.2 \%$. He was evaluated by our heart team and the decision was made to move forward with a transcatheter mitral valve replacement.

\section{Procedural techniques}

\section{Preparation}

Pre-procedural planning is key to the success of this procedure. Transesophageal echocardiography (TEE) is performed in order to confirm the severity and etiology of the mitral valve disease. Identification of endocarditis, severe paravalvular leak, bioprosthetic valve dehiscence or significant prosthesis-patient mismatch would be contraindications for a trans-septal mitral valve-in-valve ( $\mathrm{ViV})$ procedure. The essential components of pre-procedural planning include: (I) confirmation of valve sizing; and (II) identification of left ventricular outflow tract (LVOT) obstruction risk. We use a neo-LVOT area 180
$200 \mathrm{~mm}^{2}$ as the minimum area required for a mitral $\mathrm{ViV}$ procedure before we are concerned about LVOT obstruction. A high quality gated cardiac computed tomography scan is crucial for these purposes. The ViV Mitral application, is also an invaluable resource for understanding the properties of the bioprostheses (bioprosthesis height, stent internal diameter, and true internal diameter) and which transcatheter valve size can be safely implanted in a particular bioprosthesis. The reported valve size by the manufacturers are different from the measured inner stent diameters. Thus, it is important to review the dimensions of the surgical valve using the $\mathrm{ViV}$ Mitral application and to confirm the valve size using multimodality imaging in order to choose a properly sized transcatheter valve. Furthermore, this application can provide guidance when patients cannot recall their bioprosthetic valve type by displaying the fluoroscopic images of each valve type.

\section{Operation}

This procedure was performed in a hybrid operating room (OR) under general anesthesia. An arterial line was placed in the right radial artery and the procedure was performed with TEE guidance. An 8-French (Fr) sheath was inserted in the left femoral vein. A transvenous pacemaker was advanced through this sheath and positioned in the right ventricular apex. After right femoral venous access was obtained, 1 pre-close suture in the femoral vein was deployed using ProGlide (Abbott Vascular Devices, Santa Clara, California, USA) and a 16 Fr Edwards E-Sheath was inserted to accommodate the $29 \mathrm{~mm}$ valve size. After an initial 3,000 international units (IU) heparin bolus, a trans-septal puncture was performed under TEE guidance 
in a "high" and "posterior" position in the fossa ovalis. The Baylis sheath was advanced into the left atrium and an additional bolus of heparin was administered for a total of $100 \mathrm{IU} / \mathrm{kg}$ [target activated clotting time (ACT) 250-300 s]. An Agilis ${ }^{\mathrm{TM}}$ catheter (St. Jude Medical, St. Paul, MN, USA) was advanced over a ProTrack pigtail wire (Baylis Medical, Montreal, QC, Canada) into the left atrium and directed over the mitral valve. A $5 \mathrm{Fr}$ Multipurpose catheter (Cook Medical, Bloomington, IN, USA) was advanced over a standard 0.035 " guidewire through the Agilis catheter. Using TEE and fluoroscopy for guidance, the multipurpose catheter was advanced across the valve into the $\mathrm{LV}$ apex. The standard guidewire was then exchanged for a 0.035 " Confida Wire (Medtronic, Minneapolis, MN, USA) which was positioned in the LV apex. Getting adequate and stable guidewire position is crucial to a successful procedure. Subsequently, the interatrial septum was subsequently dilated with a 10-12 mm peripheral balloon through the Agilis sheath and "flossed" across the septum to ensure adequate space for the transcatheter delivery system to cross the septum. The Agilis sheath and balloon were then removed. A SAPIEN3 valve was mounted for antegrade implantation and the delivery was advanced into the inferior vena cava. This is an important step to ensure that the SAPIEN3 valve is mounted 180 degrees from the orientation typically used for transcatheter aortic valve replacement (TAVR) procedures; an inappropriately mounted valve would have dire consequences. The valve was docked and aligned on the deployment balloon in the inferior vena cava before being advanced across the atrial septum. The transcatheter valve deployment system was flexed and oriented towards the mitral valve and positioned across the mitral valve prosthesis in a fluoroscopic view perpendicular to the bioprosthesis and confirmed with TEE. It is important to obtain coaxial alignment with the bioprosthesis. The atrial aspect of the transcatheter valve should overlap with the bioprosthetic sewing ring-each valve appears has a unique fluoroscopic appearance and it is imperative for operators to know the exact location of the sewing ring on which a sealing zone is created. The objective is to have approximately $20 \%$ of the transcatheter valve in the left atrium and $80 \%$ in the left ventricle. The valve was deployed with slow balloon inflation under rapid ventricular pacing at 180-200 bpm. Slow balloon inflation allows for real-time adjustment of the transcatheter valve during deployment. The final position was assessed fluoroscopically and with TEE.

\section{Completion}

After valve deployment, the transcatheter valve deployment system was removed. A multipurpose catheter was then advanced into the $\mathrm{LV}$ to allow atraumatic removal of the Confida wire. The sheath was then removed and the right femoral venous Perclose was secured. In this case, protamine was administered, the transvenous pacer was removed, and manual compression was held after the left femoral venous sheath was removed. The patient was monitored in the intensive care unit for a day.

\section{Comments}

The mitral valve is a highly complex apparatus composed of an annulus, leaflets, chordae tendineae and papillary muscles. Multiple transcatheter technologies have evolved in an attempt to tackle functional and degenerative mitral valve pathologies in high risk surgical patients. Re-operation for patients with failed mitral bioprosthesis carries a shortterm mortality risk between $8-12 \%$ (1). Thus, transcatheter mitral valve in valve implantation with a transcatheter aortic valve prosthesis is an appealing alternative in the treatment of these patients and has been successfully used to treat patient with prior open-heart mitral valve replacements. This has been mostly performed with the Edwards SAPIEN valves (Irvine, California, USA) via either transapical or trans-septal approaches. The trans-apical approach via a mini left thoracotomy allows for relative ease in achieving coaxial alignment of the transcatheter valve to the bioprosthesis. However, when feasible, the trans-septal approach is more appealing because it offers greater technical ease and less invasiveness compared to the transapical approach in high risk patients.

Transcatheter trans-septal mitral valve implantation using transcatheter aortic valve prosthesis is a promising option in high risk redo surgical patients. This procedure was first reported in 2010 and rigorous data has been limited (2). However, there have been several small observational studies demonstrating its short-term success. One recent study involving 6 centers reported the outcomes of 60 patients with failing mitral surgical bioprostheses, 16 patients with mitral valve annuloplasty rings and 12 patients with native mitral annular calcification (3). The mean STS score was $13 \pm 8 \%$. A trans-septal approach was used in $85 \%$ of patients. Procedural success was achieved in 58 of the 60 (97\%) ViV patients. A total of 30-day and 1-year survival was acceptable in this group (95\% and 86\%, respectively). 
These results suggest that this procedure is feasible and a reasonable option for patients who require treatment for their failing surgical mitral valve bioprostheses who are deemed high risk surgical candidates by a heart team. appropriate case planning using gated computed tomography can help identify patients with suitable anatomy. In addition, from the transcatheter mitral valve replacement registry, 176 patients underwent mitral ViV implantation. Overall technical success was $96 \%$. Regarding complications, 4\% developed LVOT obstruction, 2\% suffered from a valve embolization and $1 \%$ had a left ventricular perforation (4). As these are the main risks of the procedure, it is crucial to carefully evaluate the MV prosthesis size and patient's risk of LVOT obstruction prior to the procedure. During the procedure, it is important to achieve and maintain adequate wire position in the LV.

Overall, studies suggest that these patients could benefit from this therapy in the short term. Additional long-term data in larger series are needed in order to determine the durability and long-term results of this therapy.

\section{Acknowledgements}

None.

Cite this article as: $\mathrm{Ng}$ VG, Kodali S, George I. Transcatheter trans-septal mitral valve-in-valve implantation. Ann Cardiothorac Surg 2018;7(6):821-823. doi: 10.21037/ acs.2018.09.04

\section{Footnote}

Conflicts of Interest: The authors have no conflicts of interest to declare.

\section{References}

1. Vohra HA, Whistance RN, Roubelakis A, et al. Outcome after redo-mitral valve replacement in adult patients: a 10year single-centre experience. Interact Cardiovasc Thorac Surg 2012;14:575-9.

2. Webb JG, Wood DA, Ye J, et al. Transcatheter valve-invalve implantation for failed bioprosthetic heart valves. Circulation 2010;121:1848-57.

3. Eleid MF, Whisenant BK, Cabalka AK, et al. Early Outcomes of Percutaneous Transvenous Transseptal Transcatheter Valve Implantation in Failed Bioprosthetic Mitral Valves, Ring Annuloplasty, and Severe Mitral Annular Calcification. JACC Cardiovasc Interv 2017;10:1932-42.

4. Yoon SH, Whisenant BK, Bleiziffer S, et al. Transcatheter Mitral Valve Replacement for Degenerated Bioprosthetic Valves and Failed Annuloplasty Rings. J Am Coll Cardiol 2017;70:1121-31. 